\title{
Norois
}

Environnement, aménagement, société

\section{Le conflit de Notre-Dame-des-Landes: les terres agricoles, entre réalités agraires et utopies foncières}

Farmland and the Notre-Dame-des-Landes Conflict: From Utopian Ownership Models to Agricultural Realities

\section{Cécile Rialland-Juin}

\section{OpenEdition \\ Journals}

Édition électronique

URL : http://journals.openedition.org/norois/5907

DOI : $10.4000 /$ norois. 5907

ISBN : 978-2-7535-5227-2

ISSN : $1760-8546$

\section{Éditeur}

Presses universitaires de Rennes

\section{Édition imprimée}

Date de publication : 17 octobre 2016

Pagination : 133-145

ISBN : 978-2-7535-5222-7

ISSN : 0029-182X

\section{Référence électronique}

Cécile Rialland-Juin, « Le conflit de Notre-Dame-des-Landes: les terres agricoles, entre réalités agraires et utopies foncières », Norois [En ligne], 238-239 | 2016, mis en ligne le 17 octobre 2018, consulté le 01 mai 2019. URL : http://journals.openedition.org/norois/5907 ; DOI : 10.4000/norois.5907

(c) Tous droits réservés 


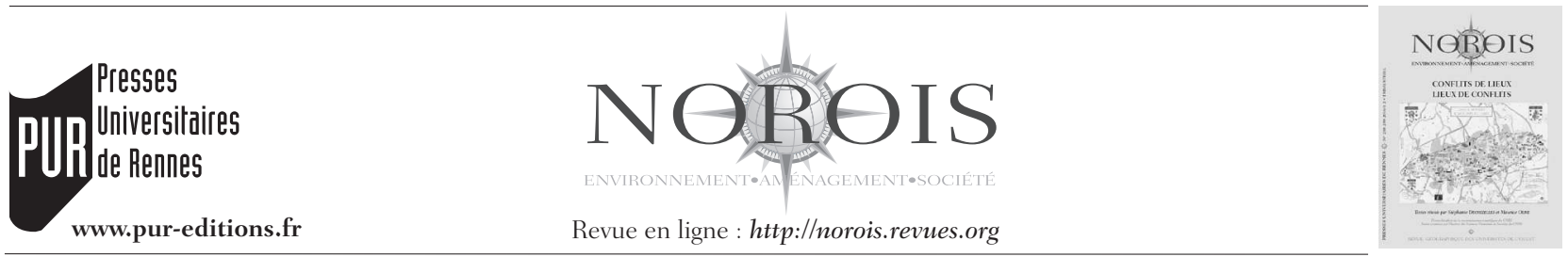

\title{
Note de recherche \\ Le conflit de Notre-Dame-des-Landes : \\ les terres agricoles, entre réalités agraires et utopies foncières
}

\author{
Farmland and the Notre-Dame-des-Landes Conflict: \\ From Utopian Ownership Models to Agricultural Realities
}

\author{
Cécile Rialland-Juin*
}

\begin{abstract}
LETG-LEESA Angers, UMR 6554 CNRS - 11 boulevard Lavoisier, 49045 AngERs cedex 01, France. (cecile. rialland-juin@univ-angers.fr)

* Élue municipale jusqu'en 2014 de la commune d'Héric, limitrophe à celle de Notre-Dame-des-Landes ; depuis 2009 membre du collectif d'élus qui doute de la pertinence du projet d'aéroport (CéDpa)
\end{abstract}

Résumé : Depuis 1974, les promoteurs du projet d'aéroport du Grand Ouest ont réservé une zone d'aménagement différé (ZAD) de 1650 hectares autour de la commune de Notre-Dame-des-Landes, à une vingtaine de kilomètres au nord de l'agglomération nantaise. Près de quarante années ont passé et, après une série de rebonds, le projet aéroportuaire est rentré dans sa phase opérationnelle lors de la déclaration de son utilité publique en 2008. Il s'est alors heurté à une amplification et radicalisation du mouvement d'opposition. Près de 300 personnes issues de mouvements alternatifs occupent alors illégalement la ZAD et la transforment en « zone à défendre » (ZAD). Au-delà du lieu d'affrontements largement médiatisé, l'espace est devenu entre-temps un véritable creuset d'expérimentations sociales, économiques et politiques. Parmi ces expériences, nous nous sommes particulièrement intéressés à la question des terres agricoles. Porteuses de dynamiques paysagères, les questions autour du foncier agraire sont au cœur d'enjeux socio-économiques et territoriaux. Nous proposons ici une lecture géographique de leurs trajectoires à différentes échelles spatiales et temporelles.

\begin{abstract}
Since 1974, the instigators of a project for a new "Great Western Airport" have earmarked land covering some 1,650 hectares (4,080 acres) around the village of Notre-Dame-des-Landes, around 20 kilometers (12 miles) to the north of Nantes, as a zone d'aménagement différé (ZAD), or "area for future development". Almost 40 years on, after a series of twists, the airport project finally entered into its operational phase following the declaration of public utility issued in 2008. At this point, the project came up against a heightening and radicalization of the opposition movement. Nearly 300 people from alternative movements illegally occupied the ZAD and transformed it into a different kind of "ZAD" - a zone à défendre, or "area to be defended". Although the area is known for being the location of widely publicized confrontations, it has at the same time also become a true melting pot of social, economic and political experimentation. Among these experiments, we have taken a particular interest in the themes relating to agricultural land. Issues surrounding agrarian land cultivation and reform - bringing to the fore ideas relating to landscape dynamics - lie at the heart of socio-economic and territorial challenges. Here, we present a geographical interpretation of their trajectories at various spatial and temporal scales.
\end{abstract}

Mots clés : terre agricole - foncier - Notre-Dame-des-Landes

Keywords: farmland - agricultural land - land ownership - Notre-Dame-des-Landes 


\section{INTRODUCTION}

Le projet d'aéroport de Notre-Dame-des-Landes est une vieille histoire. Il a pour origine l'ambitieuse politique d'aménagement du territoire initiée dans les années 1960 par la DATAR ${ }^{1}$, avec la mise en place des "métropoles d'équilibre » capables de contrer le "surpoids » de Paris. Aussi, pour accompagner le développement de l'aire métropolitaine de Nantes-Saint-Nazaire, l'idée d'un grand aéroport à vocation internationale, voire intercontinentale, voit le jour. L'aéroport de Château-Bougon (l'actuel aéroport Nantes-Atlantique), implanté depuis l'entre-deux guerres à seulement $3 \mathrm{~km}$ au sud-ouest de la ville, avec son trafic ne dépassant pas 200000 passagers, ne saurait suffire. Au bout de trois ans d'études (1965-1968), les services de la préfecture de Loire-Atlantique retiennent le site de Notre-Dame-des-Landes. L'arrêté de création de la Zone d'Aménagement Différé (ZAD) aéroportuaire, en date du 11 janvier 1974, s'applique alors à 1200 ha répartis sur 4 communes, dont l'essentiel sur le territoire de Notre-Dame-desLandes (figure 1). Au fil du temps, le projet voit ses logiques se modifier : aéroport international, aéroport pour le Concorde, aéroport de fret, puis troisième aéroport du Grand Paris, et aujourd'hui Aéroport du Grand Ouest.

Le futur aéroport est déclaré d'utilité publique en 2008, puis concédé par l'État à l'entreprise Aéroports du Grand Ouest (AGO), filiale de Vinci pour 55 ans. Le projet aéroportuaire dont l'inauguration est prévue en 2017 entre dans sa phase opérationnelle et se heurte alors à une opposition croissante. Les premières manifestations d'envergure ont lieu à l'automne 2012 lorsque les pouvoirs publics tentent d'évacuer du secteur préempté des militants altermondialistes progressivement établis suite au Plan Action Climat tenu sur la ZAD de NotreDame-des-Landes en août 2009. Rassemblés sous le terme générique de "Zadistes », ces personnes, en rupture avec le modèle de société capitaliste et opposés au projet aéroportuaire jugé emblématique des « Grands Projets Inutiles Imposés » (GPII), sont

1. Délégation à l'aménagement du territoire et à l'action régionale créée le 14 février 1963. Avec le même acronyme la DATAR désigne depuis 2009 la «Délégation interministérielle à l'aménagement du territoire et à l'attractivité régionale ». Cette administration française est chargée de préparer les orientations et de mettre en œuvre la politique nationale d'aménagement et de développement du territoire. motivées par la mise en pratique d'un mode de vie alternatif et de décroissance (Mélé, 2014; Pailloux, 2015). Les autorités publiques estiment jusqu’à 300 le nombre d'individus qui occupe de manière illégale la ZAD renommée par ces derniers « zone à défendre » (ZAD) afin d'empêcher dans l'immédiat le début du chantier à l'aide de cabanes, camions ou squats.

Depuis 1974, l'emprise et le contenu de la ZAD n'ont cessé d'évoluer selon les conjonctures économiques et sociales. Un bilan quantitatif et qualitatif du secteur, des acteurs et usages en présence s'impose pour mieux en cerner les enjeux et perspectives. Que se passe-t-il exactement avec les 1650 hectares de la Zone d'Aménagement Différé (ZAD) du projet aéroportuaire? Comment, autour du foncier, est-on passé d'une «zone d'aménagement différé " à une "zone à défendre " ? Quels sont les usages et les modes d'appropriation, légaux ou non? Quels sont les outils mobilisés et les actions menées pour assurer le maintien des terres agricoles et selon quel modèle de développement ? Enfin, si l'Aéroport du Grand Ouest ne voyait pas le jour, quelles sont les hypothèses avancées par les opposants pour gérer « l'après Notre-Dame-DesLandes »?

La bibliographie existante sur ce projet controversé est très riche. Cette note de recherche complète un corpus documentaire déjà réalisé par nos soins en 2014 (Renard et Rialland-Juin C., 2013), ainsi qu'une communication ayant pour cadre les séminaires «Conflits et Territoires » organisés et diffusés en ligne par AgroParisTech. Ces deux premières publications s'attachaient à présenter un conflit d'aménagement exemplaire par son épaisseur historique et la complexité des acteurs et enjeux en présence. Depuis le printemps 2014, plusieurs initiatives menées au sein de la ZAD autour de la question foncière ont cependant motivé de nouvelles recherches. En effet, malgré les expropriations engagées depuis les années 1970, la ZAD est restée largement occupée et exploitée par des agriculteurs aux statuts variés, rejoints plus récemment par les «Zadistes », ces derniers, pour la plupart illégaux, se sont progressivement établis sur la ZAD et entendent désormais y rester.

Dans cette perspective, le devenir des terres agricoles jusqu'ici gelées par le projet aéroportuaire fait désormais l'objet de rencontres et de débats, et suite 


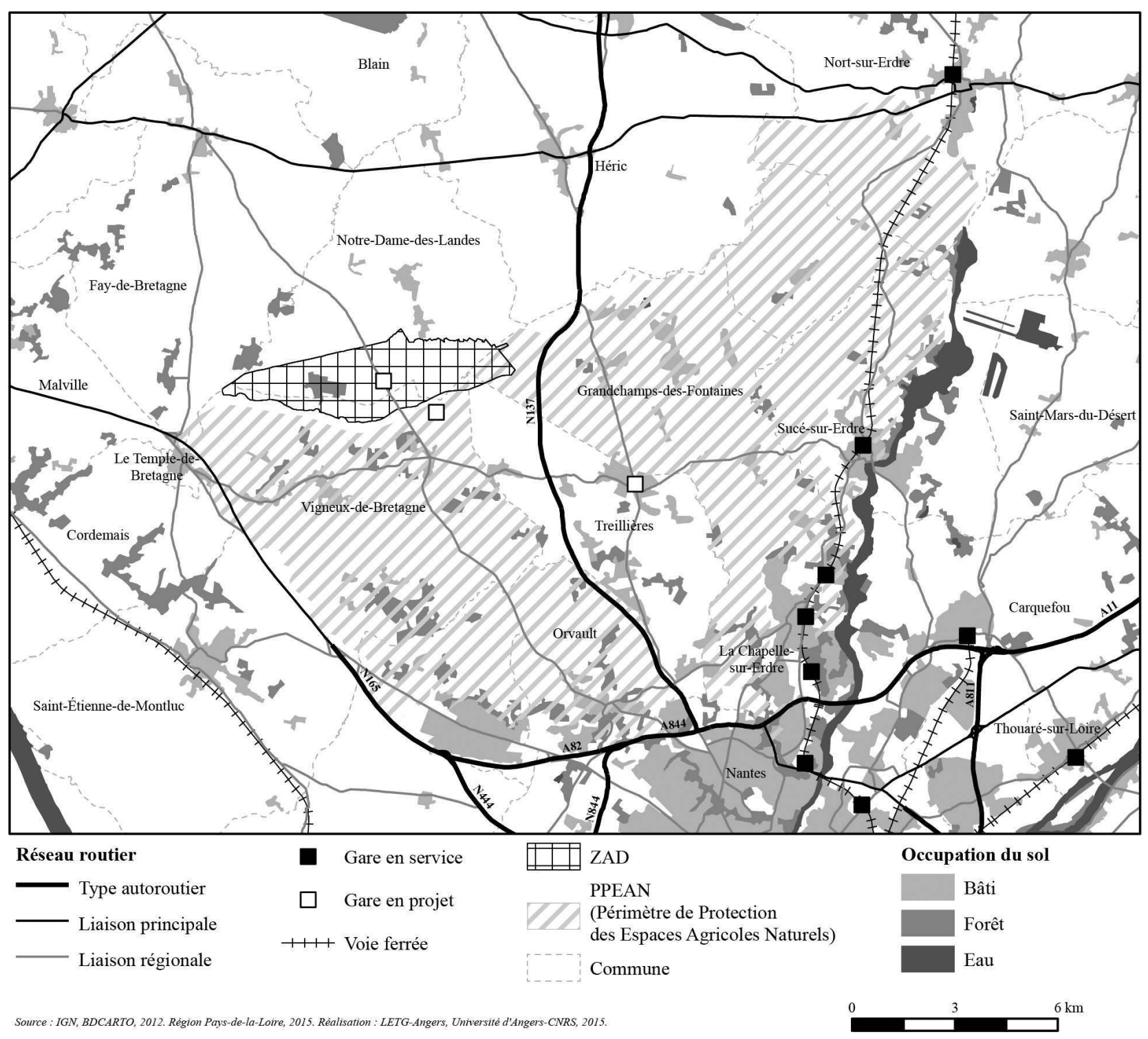

Figure 1 : La Zone d'Aménagement Différé (ZAD) du projet aéroportuaire de Notre-Dame-des-Landes The ZAD (area for future development) earmarked for the Notre-Dame-des-Landes airport project

à ces démarches, un collectif d'opposants ${ }^{2}$ rassemblant des agriculteurs et occupants de la «zone à défendre » publie en mars 2015 un état des lieux du foncier de la ZAD ${ }^{3}$. Ce document extrêmement

2. ADECA (Association de Défense des Exploitants Concernés par l'Aéroport - création 1972) - ACIPA (Association Citoyenne Intercommunale des Populations concernées par le Projet d'Aéroport, création novembre 2000, 3500 membres) -, COPAIN 44 (Collectif des Organisations Professionnelles Agricoles INdignées par le projet d'aéroports), création novembre 2012 - Occupant(e)s de la Zone à Défendre (300 personnes environ).

3. Occupant-e-s et paysan-ne-s de la zad, lu par ACIPA et COPAIN 44, 2015, Foncier - un état des lieux -. Pour penser l'avenir de la zad de Notre Dame des Landes, [https://www.acipandl.fr/images/PDF/Divers/2015/foncierzadfinalrefait.pdf]. technique et concis dresse, à partir d'informations rassemblées par les agriculteurs et occupants de la ZAD, un bilan chiffré des surfaces expropriées, occupées et/ou exploitées. Il s'appuie en partie sur une cartographie réalisée au 1/25000 par un «collectif de cartographes anonymes ». Ces derniers alimentent régulièrement un ensemble de cartes mises en ligne sur le site web de la «zone à défendre ${ }^{4}$ ». Cette cartographie, élaborée dans premier temps pour guider et informer le public des actions menées

4. Site web des occupants de la ZAD: [http://zad.nadir.org/]. 
lors des premières grandes manifestations en 2012, repose désormais sur un système d'information géographique permettant entre autres le suivi parcellaire du secteur préempté. L'intervention d'associations environnementalistes, soucieuses de fournir une contre-expertise face à celles des promoteurs du projet aéroportuaire par l'inventaire et le suivi des espèces du secteur préempté, a largement contribué à l'enrichissement des bases de données localisées. Ainsi le collectif de cartographes se réunit régulièrement pour répondre aux besoins des opposants à l'instar de ce que proposerait une structure de type « observatoire ». C'est grâce à ces travaux et en lien avec eux, que nous sommes en mesure de présenter la carte de l'occupation des parcelles de la zone d'aménagement différé (figure 2 - planche VIII), centrale à notre étude.

Outre la mobilisation des données produites par les collectifs d'opposants, l'analyse s'appuie également sur l'expérience de l'auteur comme élue municipale de la commune d'Héric, voisine de Notre-Dame-des-Landes, et membre depuis 2009 $\mathrm{du}$ «Collectif des élus qui doutent de la pertinence du projet d'aéroport » (CéDpa). Ce dernier regroupe près d'un millier d'élus de toutes familles politiques. Il a initié plusieurs « contre-expertises » et proposé des alternatives au projet aéroportuaire avec notamment l'amélioration du site actuel de « NantesAtlantique »(Verchère, 2016). Cette proximité permet une observation au quotidien des réalités du terrain. À cette expérience personnelle, on ajoutera enfin plusieurs rencontres avec les acteurs locaux à l'occasion d'excursions organisées pour des étudiants en géographie ou en sciences politiques.

Ce regard d'une géographe, porté au quotidien sur le lieu du conflit, propose un bilan des usages, acteurs et discours autour de la question foncière de l'emprise. Il précise et complète les analyses des chercheurs en géographie, sciences politiques, juridiques et économiques sur les conflits d'aménagement.

\section{DES CAMPAGNES BOCAGÈRES SOUS PRESSION URBAINE}

Vus du ciel, les paysages agraires de Notre-Damedes-Landes se caractérisent par une trame bocagère dominante, associée à de faibles densités de population périurbaine. Les formes d'occupation sont variées, mais suffisamment structurées et perma- nentes pour faire partie des réalités paysagères. Le survol du secteur préempté permet de distinguer les éléments suivants : trois sièges d'exploitations en activité malgré les expropriations (figure 3), les terres cultivées d'une dizaine d'exploitations dont le siège est en dehors de la ZAD, (prairies bocagères, cultures fourragères et troupeaux de bovins); une quinzaine de squats de "Zadistes » établis sur d'anciens sièges d'exploitation avec des jardins collectifs attenants (figure 4, figure 5) et enfin une vingtaine de cabanes (figure 6) construites par les «Zadistes » et concentrées au sud de la ZAD sur les lieux exposés aux premiers travaux du projet aéroportuaire.

Le paysage associé à la « zone à défendre » en partie initié par le mouvement altermondialiste ne s'est pas écrit sur une page blanche. Ce dernier interroge les héritages fonciers et les facteurs d'évolution socio-économiques du lieu (Renard, 1975; Croix, 1999). Jusqu'au XIX ${ }^{\mathrm{e}}$ siècle, la lande occupe la majeure partie du plateau nantais (Palierne, 1971). Au moment de la révolution industrielle, l'élite locale investit dans la terre. Elle permet une première révolution agricole et la promotion sociale de toute une paysannerie (Bourrigaud, 1994). Plus tard, dans les années 1960, les héritiers de ces exploitations, modernes avant l'heure, seront comme dans tout l'Ouest, le fer de lance du syndicalisme agricole catholique mais aussi allié à la classe ouvrière de Nantes et de Saint-Nazaire (Lambert, 1970). Les premiers opposants au projet d'aéroport unis dans l'ADECA seront des «paysans-travailleurs » en lutte contre les « grands aménageurs publics » (De Legge et Leguen, 1976).

L'épaisseur historique de ce conflit d'aménagement participe à sa singularité (Renard et RiallandJuin, 2014). Né des Trente Glorieuses et contemporain du conflit du Larzac, il connaît trois périodes : les années 1970 avec les premières luttes paysannes face à un projet d'aménagement imposé; les années 1980 avec sa mise en veille suite aux deux chocs pétroliers qui laissent penser que le projet est abandonné et favorise l'étalement urbain; les années 2000 avec la relance du projet et la radicalisation de l'opposition depuis la déclaration d'utilité publique en 2008. On assiste alors au glissement progressif de la conflictualité où des «paysans-travailleurs » entendaient défendre leur outil de travail face aux " grands aménageurs », à celui d'une une société civile, toutes catégories confondues, qui se mobilise 
Figure 3 : Le siège d'exploitation « Les Domaines », un GAEC polyculture élevage bovin lait-viande au cœur d'un bocage géométrique mis en place à la fin du $\mathrm{XIX}^{\mathrm{e}}$ siècle

L'exploitation fait l'objet d'une expropriation/expulsion. Un des hangars renommé « la Vache Rit » est un des lieux de rassemblement des opposants (cliché : Paul Bernard, mars 2015).

The main farm within the GAEC (farming collective) of "Les Domaines", specialized in beef and milk production, at the heart of a geometric field system of mixed woodland and pasture established in the late $19^{\text {th }}$ century.

The farm is the subject of an expropriation/eviction process. One of the barns, renamed from "La Vacherie" ("The Cow House") to "La Vache Rit" ("The Cow Laughs"), is one of the airport opponents' gathering places

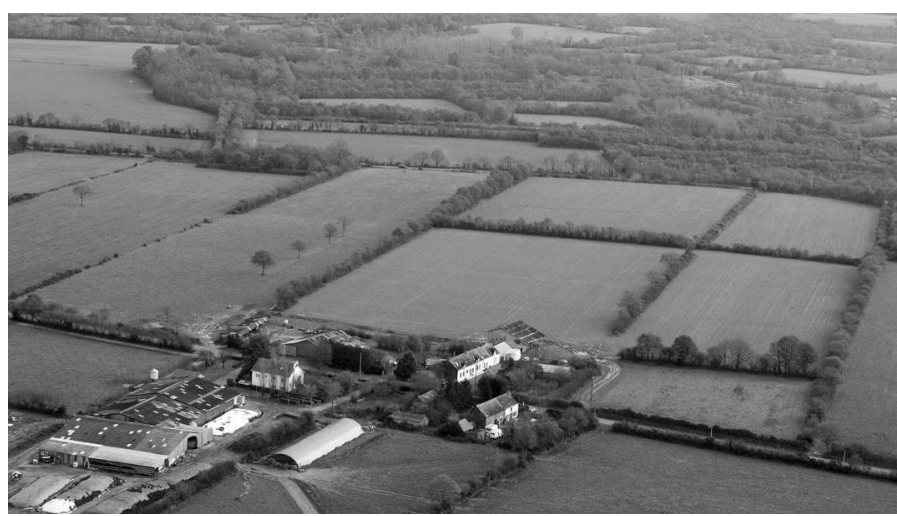

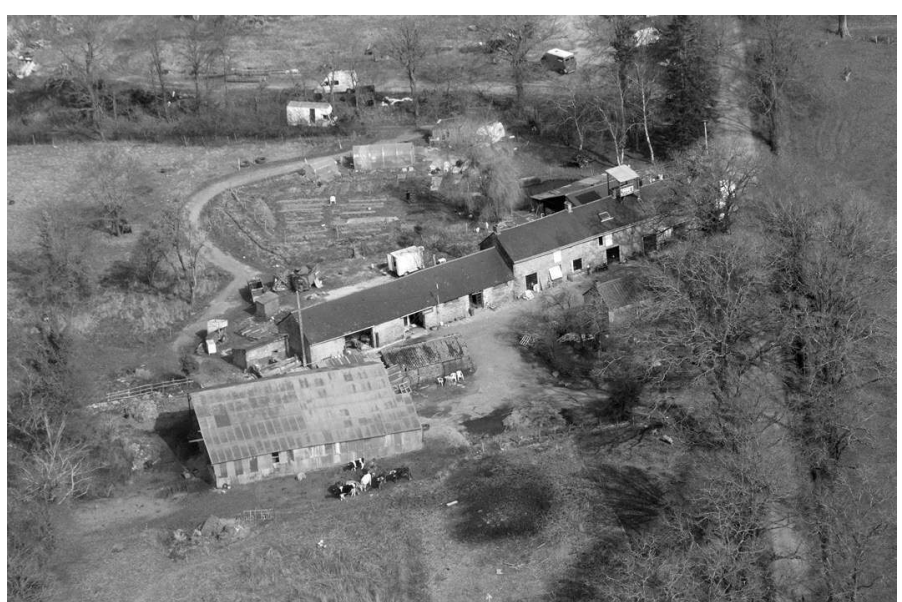

Figure 4 : L'ancienne exploitation de Bellevue expropriée et réoccupée en 2013 par le mouvement d'opposition ("Zadistes », COPAIN 44, ADECA, sympathisants ACIPA solidarités) (cliché : Paul Bernard, mars 2015)

Il s'agit d'une exploitation vivrière avec une vingtaine de bovins lait-viande donnés par des agriculteurs solidaires, des jardins collectifs et 40 hectares attenants cultivés suite aux manifestations "Sème ta ZAD » (foins, sarrasin, céréales, pommes de terre, etc.). Les récoltes sont destinées à l'autoconsommation et à des actions de solidarité.

The former Bellevue farm, expropriated and reoccupied in 2013 by the opposition movement ("Zadistes", COPAIN 44, ADECA, ACIPA supporters)

This is a food operation with 20 cattle donated by sympathetic farmers and raised for meat and milk, community gardens, and 40 hectares of land cultivated as a result of the "Sème ta ZAD" (Sow your ZAD") events (hay, buckwheat, cereal, potatoes, etc.). The crops grown are for own consumption and community-focused initiatives.
Figure 5: "Les Fosses Noires », une exploitation expropriée et occupée qui est le siège d'une boulangerie et de jardins collectifs; un « non marché » hebdomadaire (à prix libres) se tient à proximité (cliché : Paul Bernard, mars 2015)

"Les Fosses Noires", an expropriated and occupied farm that is now home to a bakery and collective gardens; a weekly "non-market" (where customers pay however much or little they like) takes place nearby

pour défendre un mode de développement durable du territoire (Subra, 2007).

À l'ombre de la métropole nantaise, les campagnes sont largement gagnées par l'étalement urbain. Le recul des terres agricoles y est relativement important. Dans un contexte de périurbanisation, le projet d'aéroport exacerbe les tensions sur le marché foncier. Au-delà de l'emprise

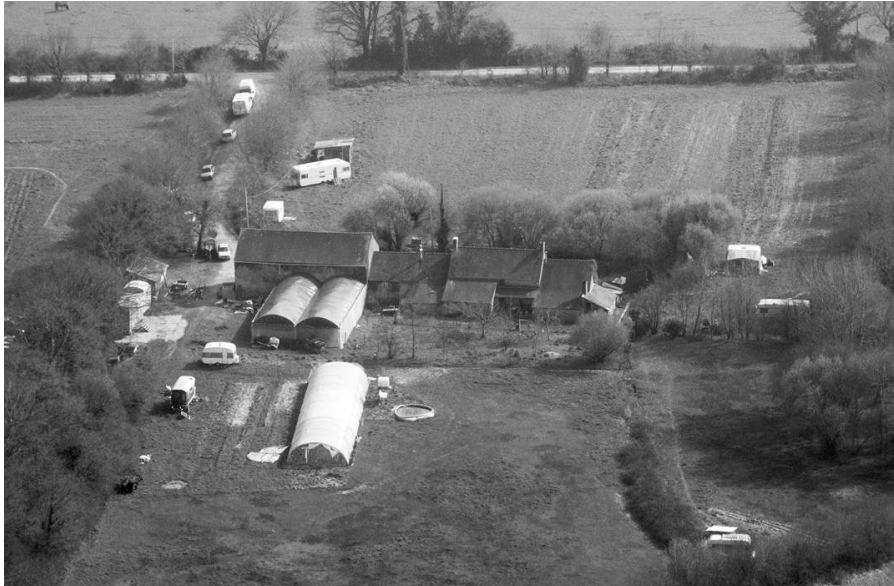

aéroportuaire, l'impact sur les surfaces agricoles restantes est important. Pour y remédier, les promoteurs du projet ont initié en 2006 la mise en place d'un Périmètre de Protection des Espaces Agricoles et Naturels ${ }^{5}$ (Margetic, 2014), visant à

5. Le PEAN est un outil relatif à la loi du Développement des Territoires Ruraux du 23 février 2005. Une vingtaine de PEAN sont en cours ou en place en France en 2015. 


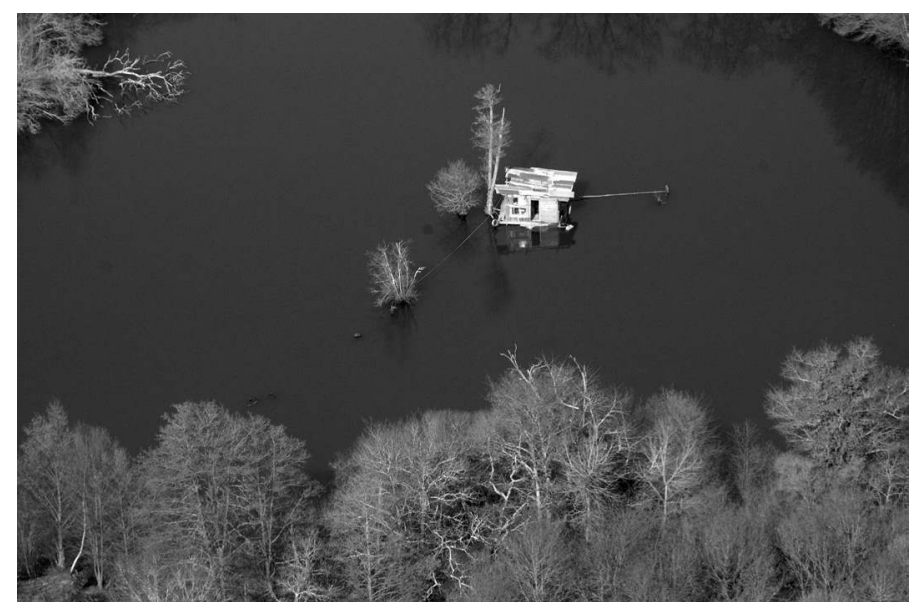

réserver à l'horizon 2025 près de 17000 hectares à l'agriculture pour éviter l'étalement urbain dans un département où la consommation de terre agricole est estimée à plus de 1500 hectares par an (Chupin et Pottier, 2011). La mise en place d'un tel outil a créé à son tour un conflit opposant cette fois les propriétaires de parcelles à chevaux menacés d'expropriation face aux exploitants agricoles (Rialland-Juin, 2012). À ce titre, la commune de Treillières, située au cœur du périmètre et soumise à une forte pression urbaine, s'est ainsi volontairement retirée du dispositif. D’autres communes comme Héric ou Fay-de-Bretagne, limitrophes du périmètre de protection, ont initié une Zone de Protection Agricole ${ }^{6}$. Même si elles restent perfectibles, ces démarches méritent cependant attention, en particulier dans un espace soumis à une forte pression urbaine et ce avec ou sans aéroport.

Outre la volonté de maintenir une quantité de terres agricoles, la qualité des sols menacés est également mise en avant. La majorité des terres réservées au projet sont des prairies humides et bocagères. Longtemps reléguées par les exigences de la modernisation agricole, elles rencontrent aujourd'hui un regain d'intérêt à la fois écologique et agronomique, confirmé par la législation environnementale européenne et nationale (Burel et Baudry, 1999).

De même, face à l'étalement de la métropole nantaise et l'artificialisation des sols induite, le

6. Zone de Protection Agricole : outil relatif à la Loi d'Orientation Agricole du 9 juillet 1999 qui permet de protéger durablement les espaces agricoles. Le classement de terrains en ZAP implique une procédure lourde pour leur changement d'utilisation, et s'impose aux documents d'urbanisme en tant que servitude d'utilité publique. L'initiative de lancer une procédure de ZAP peut être prise par les communes ou leurs groupements, mais également par le préfet.
Figure 6 : Cabane habitée du lac (cliché : Paul Bernard, mars 2015) en 2009, suite au Plan Action Climat qui s'est tenu à Notre-Damedes-Landes, près de 300 personnes liées en partie au mouvement altermondialiste ont investi la ZAD et l'ont transformé en «Zone A Défendre » à l'aide de cabanes, caravanes ou squats. Certains sites ont été détruits au moment des expulsions menées et nommées par les autorités publiques à l'automne 2012 «Opération César » . Ils ont en partie été reconstruits par les opposants quelques temps après sous le nom "d'Opération Astérix ». Inhabited shack on the lake

In 2009, following the Climate Action Plan event that was held at Notre-Dame-des-Landes, nearly 300 people, in part linked to the global justice movement, occupied and transformed the ZAD into a "zone à défendre" ("area to be defended") by setting up shacks, caravans or squats. Some sites were destroyed during the evictions carried out by the public authorities in autumn 2012, dubbed "Operation Caesar". They were partially rebuilt by opponents some time later under the code name "Operation Asterix".

droit de l'urbanisme est de plus en plus contraint par celui de l'environnement. Dans cette perspective, les campagnes au nord de Nantes, associées à des composantes naturelles à préserver (bocage, landes et zones humides en tête de bassin versant), redeviennent le support d'un mode de vie rural, plus économe en ressources et autonome qu'en ville, défendu entre autres par les occupants de la «zone à défendre » (Pailloux, 2015).

\section{UN AMÉNAGEMENT DIFFÉRÉ ET L'ÉMERGENCE D'UNE RÉSERVE FONCIÈRE}

À défaut de pouvoir construire dans l'immédiat la plateforme aéroportuaire, les promoteurs publics, par l'entremise de la chambre d'agriculture et de la DREAL ${ }^{7}$ pour les espaces naturels sensibles, ont progressivement rétrocédé près de 800 des 1650 hectares réservés sous forme de baux précaires à l'usage agricole. Au printemps 2014, c'est autour de cette réserve foncière que les opposants au projet, motivés par la sauvegarde des terres agricoles et bocagères, ont organisé des débats publics autour du statut et des modes d'utilisation des terres agricoles.

Sur les 1650 hectares de la ZAD actuelle, plus de $80 \%$ ont été acquis entre 1973 et 2003 par le conseil général de Loire-Atlantique. Outre le barreau routier (200 ha), l'ensemble de ces terres a été concédé en 2010 à la société Aéroport du Grand Ouest, (AGO), filiale de Vinci pour une durée de

\footnotetext{
7. En France les directions régionales de l'environnement, de l'aménagement et du logement (DREAL) sont les services déconcentrés du ministère de l'Environnement.
} 
55 ans. Environ 780 hectares sont encore aux mains de propriétaires privés qui refusent l'expropriation malgré la Déclaration d'Utilité Publique de 2008. Cette dernière est validée pour dix ans jusqu'en 2018, au-delà, il appartient à l'État de la prolonger ou de l'abroger.

Sur les 47 exploitations concernées par l'emprise aéroportuaire, 11 sont fortement impactées. Ces dernières, au gré des cessations d'activités des exploitants voisins, présentent des surfaces souvent supérieures à la moyenne départementale (plus de 100 hectares). Elles sont orientées vers une polyculture élevage à dominante laitière qui alimente le bassin laitier du nord de la Loire-Atlantique.

Au gré des procédures d'expropriation, d'expulsion et de démolition, mais aussi de redistribution des terres, des refus d'expropriations et de l'occupation de la ZAD, les parcelles agricoles recouvrent aujourd'hui trois réalités (figure 3) :

- 400 hectares essentiellement situés au nord de la ZAD sont cultivés au sein de quelques exploitations dites « historiques » dans la mesure où elles ont fait l'objet d'expropriations dès les années 1970 et ont, à chaque génération, refusé tout accord avec les promoteurs du projet aéroportuaire. Parmi elles, trois exploitations y ont leur siège : Les Domaines (figure 3), Liminbout et La Gennestière;

- 250 hectares sont occupés par environ trois cents «Zadistes », des alternatifs et paysans proches de la confédération paysanne (COPAIN 44). Regroupés en « collectifs », ces occupants se répartissent sur une soixantaine de lieux (fermes et habitations vouées à la destruction et habitats auto-construits). Parmi eux une dizaine de sièges d'exploitation sont aujourd'hui habités et associés à une agriculture vivrière, comme par exemple les fermes de Bellevue, des Fosses Noires ou du Haut Fay (figures 4-5-6);

- 550 hectares sont exploités par des agriculteurs qui ont bénéficié d'une redistribution de terres agricoles par l'entremise de la chambre d'agriculture sous forme de baux précaires annuels, suite à des départs en retraite d'exploitants agricoles ou de renonciation au bail contre indemnités d'éviction;

- 450 hectares enfin ne sont pas utilisés par l'agriculture, il s'agit des bois, de friches, bâtis, routes et chemins.

Malgré les opérations d'expulsion et de démolition d'une dizaine de maisons et tout autant de cabanes au sud de la ZAD menées par les pouvoirs publics entre l'automne 2012 et le printemps 2013, l'utilisation du parcellaire agricole, légale ou non, n’a cessé de se maintenir voire de se développer. Une dizaine d'exploitations poursuivent leur activité malgré les avis d'expulsion reconduits au gré des recours en justice et leur refus d'encaisser les montants d'expropriation. Les propriétaires associés à ces exploitations sont le plus souvent solidaires car l'indemnisation de 0,16 centime du $\mathrm{m}^{2}$ carré de terre agricole est jugée trop faible, surtout au regard du prix des terres constructibles du périurbain nantais qui approche les 160 euros par $\mathrm{m}^{2}$. Aussi, sur les 1650 hectares de la ZAD, 780 sont encore détenus par des propriétaires privés et 142 (18\%), ont été négociés à l'amiable depuis 2011, ce qui représente 94 propriétaires sur 577 au total (16\%). Avant cette date, le Conseil Général de Loire-Atlantique avait rétrocédé pour un euro symbolique à la société $\mathrm{AGO}$-Vinci une première réserve foncière de 870 hectares acquise dans les années soixante-dix. Ces dernières avaient également fait l'objet d'une redistribution par location aux exploitants et propriétaires expropriés.

À côté de cette stratégie d'occupation légale des exploitants-propriétaires, près de 300 personnes, issues en partie du mouvement altermondialiste, ont progressivement investi la ZAD en toute illégalité. Dans un premier temps, ces occupants ont construit des cabanes ou squatté des maisons vouées à la démolition. La tentative d'expulsions de l'automne 2012, nommée « Opération César » par les autorités publiques, a contribué à médiatiser le mouvement et par là même à le renforcer. Les cabanes et squats détruits ont été immédiatement réinvestis lors de l'opération baptisée «Astérix » par une opposition amplifiée au plan national, voire international.

À ce titre, les occupants de la ZAD ont été rejoints en 2013 par les paysans du COPAIN 44 (Collectif des Organisations Professionnelles Agricoles Indignés par le projet d'aéroport). Proche de la Confédération paysanne, et consolidé par la création de collectifs sur d'autres départements, le mouvement entend défendre et créer des emplois agricoles au sein du secteur exposé aux premiers travaux de construction du barreau routier au sud de la zone pour rendre accessible le chantier de la plateforme aéroportuaire. Ces acteurs alternatifs sont à l'origine d'une douzaine d'installations qui vont de microexploitations laitières et maraîchères à des jardins 
collectifs ou divers services visant l'autonomie progressive des communautés. Le lieu d'implantation de l'activité, quand il ne correspond pas à un toponyme, est le plus souvent rebaptisé en fonction de l'usage créé par le collectif. On peut ainsi citer : le «non-marché » hebdomadaire, un endroit, nommé « Le Gourbi », (figure 2 - planche VIII) où l'on échange les productions « made in ZAD» : pain, fromage, légumes, légumineuses, plantes médicinales, semences paysannes, etc.; la boulangerie des Fosses noires mais aussi une épicerie à prix libres, une scierie mobile ou un atelier de mécanique agricole (la « Curcuma»). Outre ces services, la ZAD possède aussi des lieux d'animations comme une maison d'accueil pour les enfants, «La smala », située à la «Wardine »; un lieu de « repas-partagé » à la Noé Verte, une radio pirate, un journal interne, un site web, une imprimerie et même une université populaire au Haut Fay pour diffuser un savoir-faire et une culture en matière de développement alternatif (figure 7).

L'occupation des bâtiments d'exploitation expropriés et ayant obtenu un permis de démolition, la mise en culture permanente des terres agricoles, la plantation de haies, sont autant d'initiatives au cœur de la stratégie des «Zadistes ». Parmi celles-ci on peut citer l'opération « Sème ta ZAD! », une manifestation qui chaque année depuis 2013 mobilise près de 400 opposants pour semer et planter une vingtaine d'hectares sur les lieux destinés à recevoir les premiers travaux. Les récoltes de foins, pommes

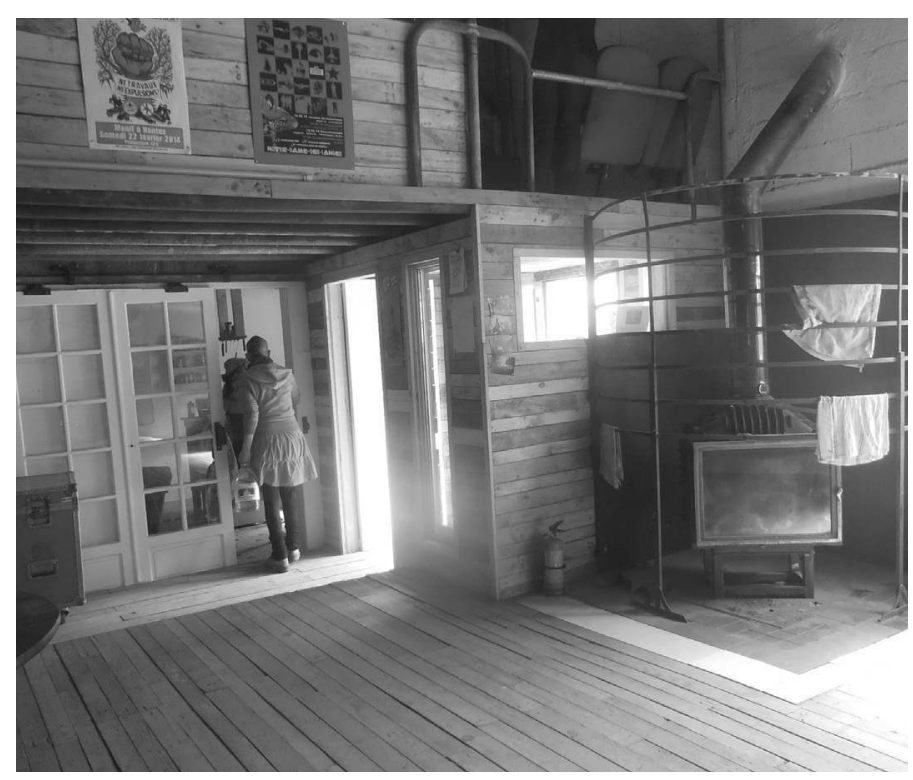

de terre, céréales ou sarrasin alimentent les communautés de la ZAD et parfois même d'autres actions de solidarité.

Proche de la ZAD, la terre fait tout autant l'objet de convoitises. Plus de $90 \%$ des sols du revers du plateau nantais y sont classés comme des zones humides bocagères situées en tête de deux bassinsversants (Vilaine et Erdre). De ce fait, la législation européenne qui ne cesse de se renforcer en faveur de la protection de l'environnement, et en particulier de l'eau, constitue une nouvelle contrainte pour les promoteurs en introduisant le principe de compensation du double des surfaces humides, haies ou mares, artificialisées par les futurs aménagements. Cette montée en puissance du droit environnemental a provoqué, autant chez les partisans que chez les opposants de l'aéroport, une ruée sur les terres agricoles situées à proximité de la ZAD.

Actuellement, les promoteurs tout comme les opposants, démarchent les propriétaires terriens ou exploitants limitrophes des secteurs préemptés afin d'acquérir des surfaces de compensation. L'objectif des premiers est d'apporter des éléments de contrepartie environnementale et les seconds de les bloquer. La bataille autour des mesures compensatoires

Figure 7 : L'Université Populaire Autonome du Haut-Fay (UPAHF) (cliché : C. Rialland-Juin, avril 2015)

Un collectif occupe depuis la fin des années 2000 la ferme expropriée du «Haut-Fay » située en limite de la ZAD sur la commune d'Héric. L'UPAHF organise des conférences et ateliers ouverts à tout public sur la mise en pratique du modèle de développement alternatif (permaculture, recyclage, autogestion, communaux, etc.). Pour « l'après Notre-Dame-des-Landes », le collectif aspire au maintien, aux côtés des Groupements Fonciers Agricoles (GFA) préconisés par les agriculteurs en lutte, des structures collectives actuelles, avec ou sans reconnaissance juridique, à l'image des « communaux des landes ", nombreux au $\mathrm{XIX}^{\mathrm{e}}$ siècle dans la région (Bourrigaud, 1994). La lande, localisée sur les sols pauvres du revers du plateau nantais, constituait pour la paysannerie locale une pâture d'appoint. Ces dernières, indivises jusqu'à la Révolution de 1789, ont progressivement été privatisées jusqu'à la fin du au XIX siècle (Kropotkine, 1909; Bourrigaud, 1994).

The Université Populaire Autonome du Haut Fay (UPAHF, or Le Haut-Fay Autonomous People's University)

Since the late 2000s, a collective has occupied the expropriated farm of Le Haut Fay, located on the edge of the ZAD near the village of Heric. The UPAHF organizes conferences and workshops open to the general public on the implementation of alternative development models (permaculture, recycling, self-management, common land, etc.). For the "post-Notre-Dame-des-Landes" period, the group aspires to maintain the current collective structures alongside the groupements fonciers agricoles (GFAs, or agricultural land groupings) advocated by activist farmers, with or without legal recognition, like the many "communaux des landes" " "heathland commoners") who lived in the region in the $19^{\text {th }}$ century (Bourrigaud, 2014). The heathland in question, located on poor soil on the slopes of the Nantes Plateau, served as extra pastureland for the local peasantry. These pastures, which remained undivided up to the French Revolution, were progressively privatized throughout the $19^{\text {th }}$ century (Kropotkin, 1909, and Bourrigand, 1994). 
passe également par une obstruction systématique de la part des « anti-aéroport » au bon déroulement des études environnementales auprès des entreprises chargées de les réaliser (interventions et destruction sur le terrain d'outils de travail, intrusions dans les locaux d'entreprises, lisibilité médiatique).

La carte des parcelles agricoles et des activités présentes dans la Zone à défendre souligne l'importance et la relative dispersion de l'occupation illégale. D’abord réticentes, voire hostiles, les populations riveraines et les agriculteurs ont progressivement admis l'efficacité de la stratégie d'implantation illégale pour empêcher le début des travaux. Progressivement, au regard des populations locales, les «Zadistes », d'abord considérés comme des marginaux et squatters d'une « zone de non droit », sont devenus des « habitants » à part entière de l'emprise aéroportuaire. En témoignent la fréquentation croissante des riverains invités aux manifestations culturelles ou ateliers initiés par les « habitants » de la ZAD. Parallèlement les échéances du projet aéroportuaire étant sans cesse reportées, les opposants locaux dont les agriculteurs en lutte, entendent soutenir et conforter la présence et les activités de ces occupants sur les 250 hectares situés au sud de la $\mathrm{ZAD}$, là où justement les premiers travaux du futur aéroport devaient permettre l'accès au chantier. La terre agricole, support des usages alternatifs, fait alors l'objet d'attentions de la part des opposants. Aussi c'est à l'occasion de rencontres au printemps 2014 entre agriculteurs en lutte, «Zadistes » et population locale que l'on dresse un premier bilan des usages et activités pour mieux en imaginer l'avenir si le projet aéroportuaire venait à être abandonné.

\section{Débats AUTOUR DU DEVENIR DES TERRES AGRICOLES DE LA ZONE D’Aménagement Différé}

Dans un contexte de report de travaux d'aménagement, des réunions publiques autour de la question du devenir des terres de Notre-Dame-des-Landes sont initiées par les occupants de la ZAD. Elles rassemblent les exploitants « historiquement » en lutte (ADECA), des « paysans-occupants » (COPAIN), les «Zadistes » (mouvement altermondialiste) et autres « citoyens-sympathisants » (ACIPA, CéDpa). Si des échanges très larges interrogent le modèle de société à développer sur ce lieu de conflits, d'autres plus pragmatiques abordent le partage et usage des terres entre les différents acteurs en présence. Suite à cette démarche, le collectif publie au printemps 2015 un premier état des lieux du foncier agricole de la ZAD.

Légalement, si la Déclaration d'Utilité Publique (DUP) de 2008 est abrogée en 2018, les propriétaires et exploitants expropriés retrouveront, s'ils le souhaitent, leur droit d'usage et de propriété. Pour le reste des terres, c'est-à-dire les 800 hectares concédés à la société AGO, les opposants s'interrogent sur leur avenir et avancent plusieurs hypothèses.

La première porte sur la mise en vente ou la location des terres à des propriétaires ou exploitants par les pouvoirs publics via la SAFER ${ }^{8}$. L'essentiel des terres redistribuées iraient alors à l'agrandissement des exploitations en place. L'après Mirabel, au Canada, témoigne d'un tel scénario (Laurin, 2012 et GagnonPoulain, 2009). Cet aéroport, situé à $40 \mathrm{~km}$ au nord de Montréal, avait été décidé par le gouvernement fédéral et inauguré en 1974. L’infrastructure a nécessité l'expropriation à bas prix de plus de 40000 hectares de terres agricoles. Conçu pour délester les vols internationaux de l'aéroport de Dorval, plus proche et contraint par la ville, l'infrastructure devait se hisser au stade de hub aéroportuaire en Amérique du Nord avec un trafic de 60 millions de passagers en 2020. Les chocs pétroliers, la concurrence avec Toronto, et la non-réalisation par le gouvernement du Québec des dessertes ferroviaires, ont eu raison de ces objectifs ambitieux. Le dernier vol de passagers a eu lieu le 30 octobre 2004 et le site est devenu une friche commerciale en attente de reconversion où subsiste un trafic de fret. L'ensemble du trafic international a été reporté sur l'aéroport de Dorval, modernisé et renommé Montréal-Trudeau en 2003. Dès 1981, le trop-plein de terres, soit plus de 30000 hectares, attenant à l'emprise aéroportuaire, est rétrocédé par l'État Fédéral aux propriétaires et exploitants. La lutte des agriculteurs, débutée en 1972, avait contribué à cette redistribution des terres, mais ces dernières sont retournées aux exploitations en place sans pour autant modifier l'état des structures : il s'agit toujours d'exploitations typiques du Québec, c'est-à-dire relativement concentrées et spécialisées en polycultureélevage.

Une seconde hypothèse repose sur la mise en place d'une entité juridique, capable de gérer collective-

8. Société d'Aménagement Foncier et d'Établissement Rural. 
ment la rétrocession des terres, afin d'éviter que la réserve foncière n'aille qu'à l'agrandissement d'exploitations individuelles. L'exemple du conflit du Larzac qui débute en 1971 contre le projet gouvernemental d'extension d'un camp militaire, est à ce titre éclairant. (Alexander-Alland, 1995). En 1981, un petit groupe de paysans soutenu par un vaste mouvement national obtient l'abandon du projet par le Président de la République François Mitterrand. En 1985, une Société Civile des Terres du Larzac (SCTL) est créée. Celle-ci organise une gestion collective de plus de 6000 hectares de terres grâce à la conclusion avec l'État d'un bail emphytéotique de 99 ans. La structure, qui rassemble tous les agriculteurs et résidents, est administrée par un conseil de gérance d'une dizaine de membres. Celle-ci attribue les exploitations, fixe le montant des baux ruraux et élabore des règles de gestion entre les différents usagers du foncier (agriculture, chasse et tourisme). En favorisant l'installation en fermage la SCTL a contribué au maintien de 25 sièges d'exploitation, à la location d'une trentaine de bâtiments pour des non-agriculteurs et à la signature d'une dizaine de baux de chasse avec des associations communales. Implantée sur 12 communes, celle-ci constitue un véritable outil de développement territorial auprès des collectivités, du Parc Naturel Régional des Grands Causses et d'autres associations civiles. Cette initiative montre qu'une gestion collective de l'usage des terres est possible à grande échelle.

Une même logique anime l'association «Terre de liens » qui, depuis 2003, mobilise les dons ou l'épargne de 1200 citoyens pour acquérir du foncier destiné à la location (2500 hectares) de quoi faciliter l'installation de plus de 150 «micro-projets » agricoles répartis sur toute la France (maraîchage biologique, paysan-boulanger, plantes aromatiques, apiculture, semences paysannes, production fromagère, fruitières, etc.).

La création d'une entité juridique de type Société Civile Immobilière (SCI), Groupement Foncier Agricole (GFA) ou autre, soulève à son tour la question de son fonctionnement, tant les acteurs en présence et les situations foncières associées sont ici multiples et variées. Aux côtés des agriculteurs « historiques » qui aspirent à habiter et à poursuivre leur activité sans être dans une précarité constante, les paysans et occupants de la ZAD entendent conserver et développer le bâti et les structures collectives qu'ils ont contribué à mettre en place. Aussi s'ac- corde-t-on à imaginer, au sein d'une entité juridique de type GFA, la coexistence de projets agricoles conventionnels aux côtés de structures collectives avec ou sans reconnaissance juridique à l'image des " communaux », largement répandus en France avant la Révolution de 1789. Il s'agit de sols souvent pauvres et à la marge du finage villageois, utilisés en commun et comme pâture d'appoint pour le bétail par la paysannerie. Ces biens communs ont progressivement disparu avec les lois révolutionnaires de 1789-1793 au profit d'une appropriation individuelle des terres (Kropotkine, 1909). Jusqu'à la fin $\mathrm{XIX}^{\mathrm{e}}$ siècle localement, on retrouve des structures de ce type avec les « communaux de lande » largement développés sur les paroisses du secteur (Bourrigaud, 1994). Pour les collectifs, l'enjeu est de préserver sans droits ni titre mais de fait près de 250 hectares au sud de la ZAD, constitués de petites exploitations vivrières, de jardins partagés et expérimentations diverses visant l'autonomie et l'utilisation parcimonieuse des ressources, où chacun entend faire valoir le bien commun sur l'intérêt privé.

\section{Conclusion}

Quarante années après la mise en place de la Zone d'Aménagement Différée, l'usage agraire des terres de Notre-Dame-des-Landes persiste, et ce malgré la volonté des promoteurs d'entamer la phase opérationnelle du projet aéroportuaire. Au-delà des actions symboliques de résistance, fortement médiatisées, le mouvement d'opposition a occasionné l'implantation progressive sur les terres de la ZAD "d'opposants d'ailleurs " aux côtés "d'opposants d'ici ». Bien que les démarches et méthodes de lutte soient différentes, chacun s'accorde à reconnaître que la «zone de non droit » des «pro-aéroport » est devenue « un lieu d'expériences collectives, de frottement de gens différents, un lieu qui donne l'image de ce qu'on peut construire ensemble ${ }^{9} »$. Face aux aspirations d'acteurs et situations foncières hétérogènes, il semble difficile d'imaginer des outils autres que variés, complémentaires et solidaires pour assurer le développement durable d'un tel territoire. Il augure d'un nouveau rapport à la terre et au-delà au territoire, plus autonome par rapport à la ville, plus économe et solidaire quant à l'exploitation des

9. Ouest France, 15 mai 2015 : «Une vie made in ZAD s'organise dans l'illégalité », propos d'un agriculteur en lutte. 
ressources (Melé, 2014). Le débat et les recherches de solutions collectives sont d'ores et déjà l'amorce d'un creuset innovant de «l'après aéroport ».

\section{Bibliographie}

Alexander-Alland A., 1995. Le Larzac et après... Étude d'un mouvement social innovateur, Paris, L'Harmattan.

Bourrigaud R., 1994. Le développement agricole an XIX siècle en Loire-Atlantique, Nantes, Éditions Centre d'histoire du travail.

Burel F., Baudry J., 1999. Écologie du paysage. Concepts, méthodes et applications, Paris, TEC \& DOC.

Chupin O., Pottier P., 2011. Dix ans d'évolution de l'occupation des sols en Loire-Atlantique (1999-2009), Cahiers Nantais, 2011/2, p. 77-87.

Croix N., 1999. La Terre, entre terroir et territoire. Mutations foncières et organisation des campagnes armoricaines méridionales (1968-1998), thèse de doctorat en géographie, Université de Nantes.

De Legge J., Leguen R., 1976. Dégage!... On aménage, Les Sables-d'Olonne, Le Cercle d'Or.

Margétic C., 2014. Les PEAN en France, des envies aux premières concrétisations, POUR no 224, p. 341-349.

Gagnon-Poulin E., 2009. La mobilisation politique des expropriés de Mirabel, Master d'Anthropologie, Université de Montréal.

Kropotine P., 1909. La Grande Révolution 1789-1793, Paris, P.-V. Stock.

Lambert B., 1970. Les paysans dans la lutte des classes, Paris, Seuil.

Laurin S., 2012. L'échiquier de Mirabel, Montréal, Éditions du Boréal.

Melé P., 2014. Du barrage de Sivens à Notre-Dame-des-Landes : extension des domaines de la lutte?, Les grands entretiens de la Mission Agrobiosciences du 18 novembre 2014, Castanet-Tolosan, éditions Mission Agrobiosciences.

Pailloux A.-L., 2015. Zone d'aménagement différé contre « zone à défendre ». Analyse d'une lutte pour l'autonomie dans/de l'espace rural, justice spatiale | spatial justice, $\mathrm{n}^{\circ} 7$, [http://www.jssj.org].

Palierne J.-M., 1971. Les fondements de l'openfield LigeroAtlantique : sols, soleil et solidarité agraire, Norois, vol. 71, p 437-449.

Renard J., 1975. Les Évolutions contemporaines de la vie rurale dans la région nantaise: Loire-Atlantique, bocages vendéens, Manges, Les Sables-d'Olonne, Le Cercle d'or.

Renard J., 2012. Les Campagnes nantaises: Un demi-siècle de révolutions sociales et paysagères (1960-2010), Rennes, PUR.

Rialland-Juin C., 2010. Analyse critique d'un outil pour la protection agricole et environnementale de l'agglomération nantaise, communication au colloque franco-britannique de géographie rurale, Université d'Essex, Colchester, Essex "Quelle agriculture pour une campagne durable? », 12- 15 juillet 2010.

Rialland-Juin C., 2012, Usages récréatifs et agriculture au nord de Nantes. Tensions autour de la mise en place d'un périmètre de protection d'espaces agricoles et naturels, Projets de Paysage, $\mathrm{n}^{\circ} 8$.

Rialland-Juin C., Renard J., 2013. Le projet d'aéroport de Notre-Dame-des-Landes : les rebonds d'un aménagement conflictuel, Géoconfluences, ENS Lyon, corpus documentaire en ligne : [http://geoconfluences.ens-lyon.fr/doc/territ/ FranceMut/FranceMutDoc17.htm].

Rialland-Juin C., 2014. Un aménagement conflictuel, le projet d'aéroport Notre Dame des Lande. SAD-APT-INRA AgroParisTech, conférence du 29 avril 2014 en ligne : [https:// www6.versailles-grignon.inra.fr/sadapt/Equipe-Proximites/ Seminaire-Conflits-et-territoires/Dernieres-interventions/ Cecile-RIALLAND-JUIN].

Subra P., 2007. Géopolitique de l'aménagement du territoire, Paris, Armand Colin.

Verchère F., 2016. Notre-Dame-des-Landes : la fabrication d'un mensonge d'État, La Colle-sur-Loup, Tim Buctu Éditions.

Le syndicat mixte d'études de l'aéroport du Grand Ouest. [http://aeroport-grandouest.fr/].

Le futur aéroport du Grand Ouest (Vinci), [www.nantes.futuraeroport.fr].

L'ACIPA, Association citoyenne intercommunale des populations concernées par le projet d'aéroport de Notre-Damedes-Landes, [http://acipa.free.fr/].

Le CéDpa, collectif d'élu-e-s doutant de la pertinence de l'aéroport, [http://aeroportnddl.fr/].

Les occupants de la ZAD, [http://zad.nadir.org/].

Terre de liens, [http://www.terredeliens.org/].

La Société Civile des Terres du Larzac, [http://www.larzac.org].

France 3 Pays de la Loire, [http://pays-de-la-loire.france3.fr/aeroport-Notre-Dame-des-Landes].

L'Express, L'aéroport, Notre-Dame-des-Landes, [www. lexpress.fr/actualite/politique/l-aeroport-Notre-Dame-desLandes_1188165.html].

Global Magazine, Notre-Dame-des-Landes, les pistes de la géographie, [www.globalmagazine.info/article/423/78/Les-pistesde-la-geographie].

Libération, Notre-Dame-des-Landes, [www liberation.fr/NotreDame-des-Landes].

Ouest France, Projet d'aéroport Notre-Dame-des-Landes, un projet qui divise, [www.onest-france.fr/dossiers/accueil-Projet-d-aeroport-Notre-Dame-des-Landes].

Le Monde, Notre-Dame-des-Landes, le projet qui divise depuis 40 ans, [www.lemonde.fr/planete/article/2012/11/16/NotreDame-des-Landes-le-projet-qui-divise-depuis-quaranteans_1791768_3244.h].

Rue 89, Notre-Dame-des-Landes, [www.rue89.com/NotreDame-des-Landes].

Kergosien C., Morin P., 2012. Notre-Dame-des-Landes, au cœur de la lutte, film documentaire, $1 \mathrm{~h} 13$ [http://lagoradebretagne.free.fr/notredamedeslandes.html].

Ferié T., 2013. Le tarmac est dans le pré, film documentaire, $55 \mathrm{mn}[$ http://pluzz.francetv.fr/videos/le_tarmac_est_dans_le_ pre_,91073272.html]. 
planche VIII (Cécile RialLAND-JUIN - Le conflit de Notre-Dame-des-Landes...)

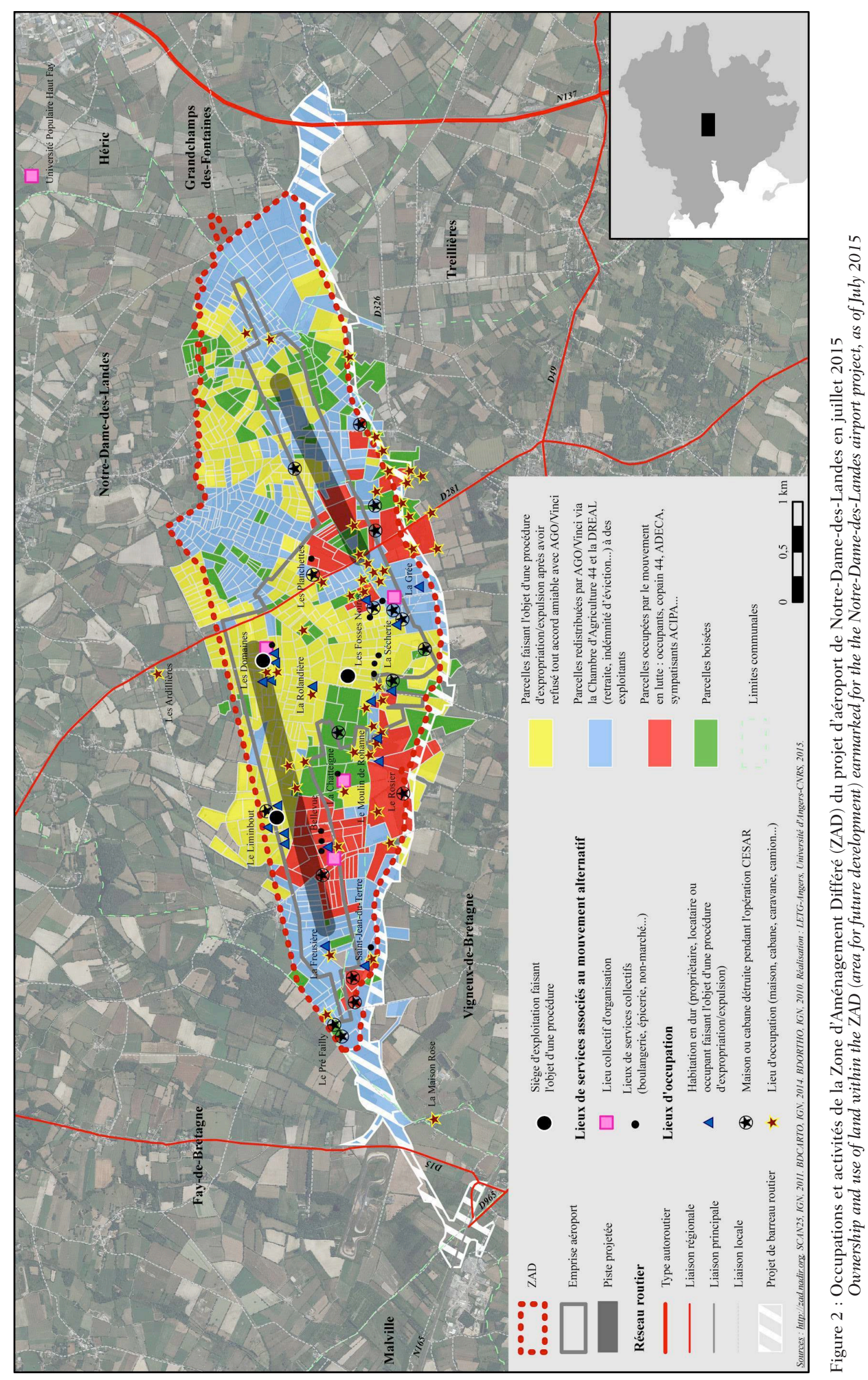

